\title{
Nuclear forces from quenched and $2+1$ flavor lattice QCD using the PACS-CS gauge configurations
}

\author{
Noriyoshi Ishii* \\ Center for Computational Sciences, University of Tsukuba, \\ Tsukuba, Ibaraki 305-8577, Japan \\ E-mail: ishii@ribf.riken.jp
}

\section{Sinya Aoki}

Graduate School of Pure and Applied Sciences, University of Tsukuba, Tsukuba, Ibaraki 305-8577, Japan

RIKEN BNL Research Center, Brookhaven National Laboratory,

Upton, New York 11973, USA

E-mail: saoki@het.ph.tsukuba.ac.jp

\section{Tetsuo Hatsuda}

Department of Physics, University of Tokyo,

Tokyo 113-0033, Japan

E-mail: hatsuda@phys.s.u-tokyo.ac.jp

\section{for PACS-CS Collaboration}

Two of recent progress in lattice QCD approach to nuclear force are reported. (i) Tensor force from quenched lattice QCD: By truncating the derivative expansion of inter-nucleon potential to the strictly local terms, we obtain central force $V_{\mathrm{C}}(r)$ and tensor force $V_{\mathrm{T}}(r)$ separately from swave and d-wave components of Bethe-Salpeter wave function for two nucleon state with $J^{P}=$ $1^{+}$. Numerical calculation is performed with quenched QCD on $32^{4}$ lattice using the standard plaquette action at $\beta=5.7$ with the standard Wilson quark action with $\kappa=0.1640,0.1665,0.1678$. Preliminary results show that the depths of the resulting tensor force amount to 20 to $40 \mathrm{MeV}$, which is enhanced in the light quark mass region. (ii) Nuclear force from $2+1$ flavor QCD with PACS-CS gauge configuration: Preliminary full QCD results are obtained by using $2+1$ flavor gauge configurations generated by PACS-CS collaboration. The resulting potential has the midium range attraction of about $30 \mathrm{MeV}$ similar to the preceding quenched calculations. However, the repulsive core at short distance is significantly stronger than the corresponding quenched QCD result.

The XXVI International Symposium on Lattice Field Theory

July 14-192008

Williamsburg, Virginia, USA

\footnotetext{
* Speaker.
} 


\section{Introduction}

Proton and neutron are the fundamental constituents of atomic nucleus. The interaction among them are called as the nuclear force. Together with the structures of nuclei, the nuclear force itself has been actively investigated in nuclear physics since its discovery about 75 yeas ago. Phenomenologically, the properties of the nuclear force are characterized by the attraction at medium distance with the OPEP tail [1,2] and the strong repulsive core [3] at short distance. The former is responsible for nuclei to be bound. The latter is important for various phenomena, such as the stability of atomic nuclei, super nova explosions of type II, and the maximum mass of neutron star. While the medium to long distance properties are accessible with the meson exchange picture, it is difficult to approach the short distance properties. In particular, the origin of the repulsive core has not yet been answered beyond phenomenological models. Since the nucleons overlaps at short distance, the repulsive core is expected to reflect the internal structure of nucleon. Therefore, QCD is considered to be the best tool to reveal the short distance properties of the nuclear force. Indeed, one attempted to study the repulsive core with lattice QCD by extending the method of static quark potential [4]. However, the repulsive core was not reproduced from this pioneering work.

Recently, we have developed a new method to extract the nuclear force between nucleons composed of non-static quarks and have found that essential features of the nuclear force are reproduced [5]. In this method, lattice QCD is used to generate the Bethe-Salpeter (BS) wave function for a two nucleon state in the center of mass frame:

$$
\psi_{\alpha \beta}(\vec{x}-\vec{y}) \equiv \lim _{t \rightarrow+0}\left\langle 0\left|T\left[p_{\alpha}(\vec{x}, t) n_{\beta}(\vec{y}, 0)\right]\right| N N\right\rangle
$$

where $p_{\alpha} \equiv \varepsilon_{a b c}\left(u_{a}^{T} C \gamma_{5} d_{b}\right) u_{c ; \alpha}$ and $n_{\beta} \equiv \varepsilon_{a b c}\left(u_{a}^{T} C \gamma_{5} d_{b}\right) d_{c ; \beta}$ denote interpolating fields for proton and neutron, respectively. Note that this represents an amplitude to find three quarks at $\vec{x}$ and other three quarks at $\vec{y}$. At large separation, i.e., $|\vec{x}-\vec{y}| \rightarrow$ large, it shows a desirable asymptotic behavior, which is characterized by the scattering phase shift $\delta(k)$ as

$$
\psi(\vec{r}) \sim \frac{\sin (k r+\delta(k))}{k r}+\cdots \quad \text { for s-wave. }
$$

Here, $k$ corresponds to the "asymptotic momentum" measured beyond the range of the interaction, which is related to the total energy $P_{0}$ of the state in Eq. (1.1) as $P_{0}=2 \sqrt{m_{\mathrm{N}}^{2}+k^{2}}$. The amplitude Eq. (1.1) satisfies the effective Schrödinger equation as

$$
\left(\vec{\nabla}^{2}+k^{2}\right) \psi(\vec{r})=m_{\mathrm{N}} \int d^{3} r^{\prime} V_{N N}\left(\vec{r}, \vec{r}^{\prime}\right) \psi\left(\vec{r}^{\prime}\right) .
$$

(For derivation, see Ref. [6].) In the r.h.s., $V_{\mathrm{NN}}$ plays the role of the interaction kernel. It is most generally non-local, and can be defined to be independent of the total energy of the state [6]. After the constraints from various symmetris are imposed, the derivative expansion leads us to

$$
V_{\mathrm{NN}}\left(\vec{r}, \vec{r}^{\prime}\right)=\left\{V_{\mathrm{C}}(r)+V_{\mathrm{T}}(r) S_{12}+V_{\mathrm{LS}}(r) \vec{L} \cdot \vec{S}+O\left(\nabla^{2}\right)\right\} \delta\left(\vec{r}-\vec{r}^{\prime}\right) .
$$

Here, $S_{12} \equiv 3\left(\vec{\sigma}_{1} \cdot \vec{r}\right)\left(\vec{\sigma}_{2} \cdot \vec{r}\right) / r^{2}-\vec{\sigma}_{1} \cdot \vec{\sigma}_{2}, \vec{L} \equiv-i \vec{r} \times \vec{\nabla}$, and $\vec{S} \equiv\left(\vec{\sigma}_{1}+\vec{\sigma}_{2}\right) / 2 . V_{\mathrm{C}}(r), V_{\mathrm{T}}(r)$ and $V_{\mathrm{LS}}(r)$ are referred to as "central force", "tensor force", and "LS force". (Iso-spin dependence of 
these potentials are understood to be implicit.) These three forces play the most important role in the conventional nuclear physics. By truncating Eq. (1.4) up to the first term, the central potential $V_{\mathrm{C}}\left(r ;{ }^{1} S_{0}\right)$ and the effective central potential $V_{\mathrm{C}}^{\text {eff }}\left(r ;{ }^{3} S_{1}\right)$ have been calculated from quenched lattice QCD [5]. The resulting potentials possess the repulsive core at short distance as well as the attraction at medium distance [5]. Note that, owing to Eq. (1.2), the method can be extended to be more faithful to NN scattering experiments [6].

The contents are organized as follows. In Sect. 2, we extend our method to tensor force, which is obtained from Schrödinger equation in coupled ${ }^{3} S-D_{1}$ partial waves in $J^{P}=1^{+}$. In Sect. 3, we give 2+1 flavor lattice QCD results of nuclear force by using PACS-CS gauge configurations.

\section{Tensor force from quenched lattice QCD}

Tensor force plays an important role in nuclear physics. Together with the repulsive core, it has important influences on the structures and the stabilities of nuclei. However, phenomenological determination of tensor force is known to be afflicted with an uncertainty especially at short distance due to the existence of centrifugal barrier.

To obtain tensor force in lattice QCD, we consider Schrödinger equation for $J^{P}=1^{+}$. In this case, the wave function has two components, i.e., s-wave component and d-wave component. The central force $V_{\mathrm{C}}(r)$ acts separately within these two components. The tensor force $V_{\mathrm{T}}(r)$ provides a coupling between these two, and the action of the LS force $V_{\mathrm{LS}}(r)$ is restricted within the d-wave component. If we keep only the first term in Eq. (1.4), we can obtain only the effective central force $V_{\mathrm{C}}^{\text {eff }}\left(r ;{ }^{3} S_{1}\right)$. By keeping the one more term in Eq. (1.4), it is possible to obtain the central force $V_{\mathrm{C}}(r)\left(\equiv V_{\mathrm{C}}\left(r ;{ }^{3} S_{1}\right)\right)$ and the tensor force $V_{\mathrm{T}}(r)$ separately. Note that these two terms give strictly local contributions in Eq. (1.4). The effective Schrödinger equation Eq. (1.3) becomes

$$
\left(H_{0}+V_{\mathrm{C}}(r)+V_{\mathrm{T}}(r) S_{12}\right) \psi(\vec{r})=E \psi(\vec{r}),
$$

where $H_{0} \equiv-\nabla^{2} / m_{\mathrm{N}}$, and $E \equiv k^{2} / m_{\mathrm{N}}$ denotes the non-relativistic energy. For definiteness, we restrict ourselves to BS wave function $\psi(\vec{r})$ for an state with the azimuthal quantum number $M=0$, i.e.,

$$
\psi_{\alpha \beta}(\vec{x}-\vec{y}) \equiv \lim _{t \rightarrow+0}\left\langle 0\left|T\left[p_{\alpha}(\vec{x}, t) n_{\beta}(\vec{y}, 0)\right]\right| N N\left(J^{P}=1^{+} ; M=0\right)\right\rangle .
$$

We define projection operators $\mathscr{P}$ and $\mathscr{Q}$ onto s-wave and d-wave components, respectively, as

$$
\mathscr{P} \psi_{\alpha \beta}(\vec{r}) \equiv \frac{1}{24} \sum_{g \in O} \psi_{\alpha \beta}\left(g^{-1} \vec{r}\right), \quad \mathscr{Q} \equiv 1-\mathscr{P} .
$$

where $O$ denotes the cubic group, which consists of 24 elements. We multiply $\mathscr{P}$ and $\mathscr{Q}$ to Eq. (2.1) from the left. Since $H_{0}, V_{\mathrm{C}}(r)$ and $V_{\mathrm{T}}(r)$ commute with $\mathscr{P}$ and $\mathscr{Q}$, Eq. (2.1) splits into the following two equations as

$$
\begin{aligned}
H_{0}[\mathscr{P} \psi](\vec{r})+V_{\mathrm{C}}(r)[\mathscr{P} \psi](\vec{r})+V_{\mathrm{T}}(r)\left[\mathscr{P} S_{12} \psi\right](\vec{r}) & =E[\mathscr{P} \psi](\vec{r}) \\
H_{0}[\mathscr{Q} \psi](\vec{r})+V_{\mathrm{C}}(r)[\mathscr{Q} \psi](\vec{r})+V_{\mathrm{T}}(r)\left[\mathscr{Q} S_{12} \psi\right](\vec{r}) & =E[\mathscr{Q} \psi](\vec{r}) .
\end{aligned}
$$

Note that each of these equations has two Dirac indices $\alpha$ and $\beta$. For definiteness, we pick up $(\alpha, \beta)=(1,0)$ components of these two equations, and solve them for $V_{\mathrm{C}}(r)$ and $V_{\mathrm{T}}(r)$. We arrive 
at

$$
\begin{aligned}
& V_{\mathrm{C}}(\vec{r})=E+\frac{1}{\Delta(\vec{r})}\left(\left[\mathscr{Q} S_{12} \psi\right](\vec{r}) H_{0}[\mathscr{P} \psi](\vec{r})-\left[\mathscr{P} S_{12} \psi\right](\vec{r}) H_{0}[\mathscr{Q} \psi](\vec{r})\right) \\
& V_{\mathrm{T}}(\vec{r})=\frac{1}{\Delta(\vec{r})}\left(-[\mathscr{Q} \psi](\vec{r}) H_{0}[\mathscr{P} \psi](\vec{r})+[\mathscr{P} \psi](\vec{r}) H_{0}[\mathscr{Q} \psi](\vec{r})\right)
\end{aligned}
$$

with $\Delta(\vec{r}) \equiv[\mathscr{P} \psi](\vec{r})\left[\mathscr{Q} S_{12} \psi\right](\vec{r})-[\mathscr{Q} \psi](\vec{r})\left[\mathscr{P} S_{12} \psi\right](\vec{r})$. Note that, if the d-wave component in the wave function vanishes, the first line in Eq. (2.6) reduces to $V_{\mathrm{C}}(r)=\left(E-H_{0}\right) \psi(\vec{r}) / \psi(\vec{r})$.

Numerical calculation is performed with quenched QCD by using Blue Gene/L at KEK. The quenched gauge configurations are generated by employing the standard plaquette gauge action at $\beta=5.7$, which leads to the lattice spacing $a^{-1}=1.44 \mathrm{GeV}$ [7]. Propagators of quarks are generated by employing the standard Wilson quark action with the hopping parameters $\kappa=$ $0.1640,0.1665,0.1678$, which correspond to $m_{\pi} \simeq 731,529,380 \mathrm{MeV}$, respectively. These calculations are performed by using $N_{\text {conf }}=1000,2000,2021$ gauge configurations. BS wave functions are picked up from the time-slice $t-t_{0}=9,8,6$, respectively, where the ground state saturation is achieved within the error bars. While the periodic boundary condition is imposed along the spatial directions, Dirichlet boundary condition is imposed along the temporal direction on the time-slice $t=0$. Wall source is used on the time-slice $t=t_{0} \equiv 5$ after imposing Coulomb gauge.

The 1.h.s. of Fig. 1 shows BS wave functions of a $J^{P}=1^{+}, M=0$ state for $m_{\pi} \simeq 529 \mathrm{MeV}$. To reduce the calculational cost, calculation is restricted to the points on the coordinate axes and their nearest neighbors for $r \gtrsim 0.7 \mathrm{fm}$, whereas all points are calculated for $r \lesssim 0.7 \mathrm{fm}$. Note that ${ }^{3} D_{1}$ part of the wave function is multivalued as a function of $r$, which is due to the angular dependence. The r.h.s. of Fig. 1 shows the resulting central force $V_{\mathrm{C}}(r)$ and tensor force $V_{\mathrm{T}}(r)$ together with effective central force $V_{\mathrm{C}}^{\text {eff }}(r) \equiv\left(E-H_{0}\right)[\mathscr{P} \psi](\vec{r}) /[\mathscr{P} \psi](\vec{r})$. From viewpoint of the meson exchange picture, this shape of $V_{\mathrm{T}}(r)$ is expected from a cancellation between the contributions from pion and rho meson. Note that $V_{\mathrm{C}}^{\text {eff }}(r)$ contains the effect of $V_{\mathrm{T}}(r)$ through the 2nd order perturbation, where one expects that $V_{\mathrm{C}}^{\text {eff }}(r)$ acquires sufficient attraction to generate the bound deuteron. However, we see that the difference between $V_{\mathrm{C}}(r)$ and $V_{\mathrm{C}}^{\text {eff }}(r)$ is quite small, which may be due to an artifact of heavy quark mass. Fig. 2 shows the quark mass dependence of tensor force $V_{\mathrm{T}}(r)$. We see that tensor force is enhanced in the light quark mass region, which suggests the importance of direct lattice QCD calculation employing light quark mass.

A technical comment is in order. Recall that we used the spin $(1,0)$ component of Eq. (2.6), where the 2 nd equation vanishes at $\vec{r} \propto( \pm 1, \pm 1, \pm 1)$. This is because the spin $(1,0)$ component of d-wave part in the wave function for $J^{P}=1^{+}, M=0$ is proportional to the spherical harmonics $Y_{M=0}^{(l=2)}(\theta, \phi) \propto 3 \cos ^{2} \theta-1$, which vanishes at $\vec{r} \propto( \pm 1, \pm 1, \pm 1)$. Although these points are removed from the plots, statistical error is accumulated in the neighborhood of these points. (For instance, see the points at $r \simeq 0.5 \mathrm{fm}$ in Figs. 1 and 2.) It is desirable to improve this in the near future.

\section{Nuclear force from $2+1$ flavor lattice $\mathbf{Q C D}$}

To compare our results with empirical data, a key role is played by a full QCD calculation on a large volume employing a smaller quark mass. PACS-CS collaboration is generating $2+1$ flavor gauge configurations on a large volume in significantly light quark mass region [9]. PACSCS gauge configurations are generated by employing Iwasaki gauge action at $\beta=1.90$ on $32^{3} \times$ 

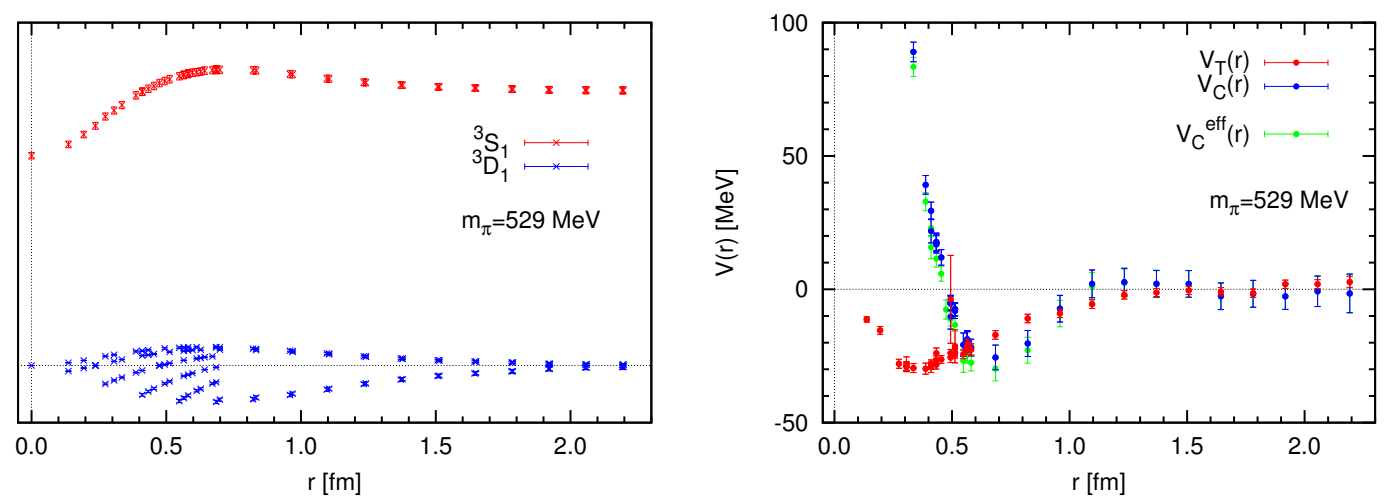

Figure 1: $(\alpha, \beta)=(1,0)$ part of the s-wave and the d-wave components of BS wave function for a state with $J^{P}=1^{+}, M=0$ (left) and the reconstructed inter-nucleon potentials $V_{\mathrm{C}}(r)$ and $V_{\mathrm{T}}(r)$ (right).

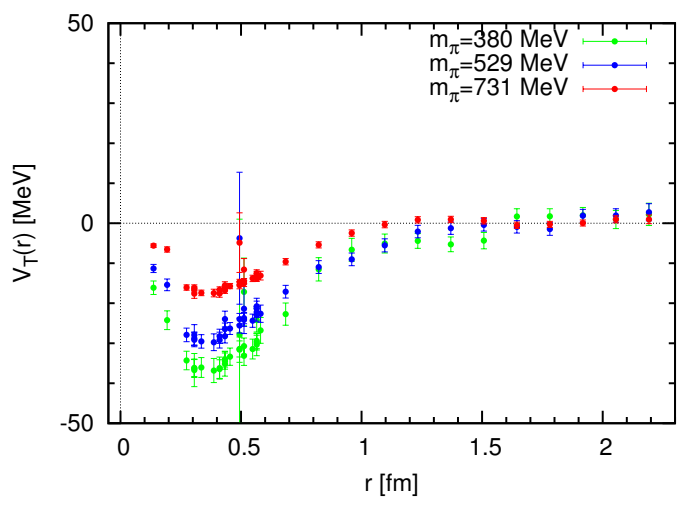

Figure 2: Quark mass dependence of tensor force.

64 lattice and $\mathrm{O}(\mathrm{a})$-improved Wilson quark (clover) action with a non-perturbatively improved coefficient $c_{\mathrm{SW}}=1.715$ [9]. The lattice scale is determined from $m_{\pi}, m_{K}$ and $m_{\Omega}$ inputs leading to $a^{-1}=2.176(31) \mathrm{GeV}(a \simeq 0.091 \mathrm{fm})$. Hence, the spatial extension amounts to $L=32 a \simeq 2.90 \mathrm{fm}$. To calculate nuclear force, we use two series of PACS-CS gauge configurations with $\left(\kappa_{\mathrm{ud}}, \kappa_{\mathrm{s}}\right)=$ $(0.13700,0.13640)$ and $(0.13770,0.13640)$, which correspond to $m_{\pi} \simeq 702,296 \mathrm{MeV}$, respectively.

To calculate BS wave function, we impose the periodic boundary condition along the spatial direction. On the other hand, along the temporal direction, we impose the Dirichlet boundary condition on the time-slice $t=32\left(=N_{t} / 2\right)$. We locate the wall source on the time-slice $t=0$ with Coulomb gauge. Note that the setup is strictly symmetric around the hyper-plane $t=0$ aiming at doubling the number of data by using the charge conjugation and the time-reversal. (See below.)

We consider the Euclidean four point correlator of nucleon fields with wall source as

$$
G_{\alpha \beta ; \alpha^{\prime} \beta^{\prime}}(\vec{x}, \vec{y}, t) \equiv\left\langle 0\left|T\left[p_{\alpha}(\vec{x}, t) n_{\beta}(\vec{y}, t) \bar{p}_{\alpha^{\prime}}^{\prime} \bar{n}_{\beta^{\prime}}^{\prime}\right]\right| 0\right\rangle,
$$

where $\bar{p}_{\alpha}^{\prime} \equiv \sum_{\vec{x}, \vec{y}, \vec{z}} \varepsilon_{a b c}\left(\bar{u}_{a}(\vec{x}) C \gamma_{5} \bar{d}_{b}^{T}(\vec{y})\right) \bar{u}_{c ; \alpha}(\vec{z})$ and $\bar{n}_{\beta}^{\prime} \equiv \sum_{\vec{x}, \vec{y}, \vec{z}} \varepsilon_{a b c}\left(\bar{u}_{a}(\vec{x}) C \gamma_{5} \bar{d}_{b}^{T}(\vec{y})\right) \bar{d}_{d ; \beta}(\vec{z})$ denote wall sources for proton and neutron, respectively. Statistical noises are reduced by utilizing the 
following four symmetries. (i) The spatial translation: $G_{\alpha \beta ; \alpha^{\prime} \beta^{\prime}}(\vec{x}, \vec{y}, t)=G_{\alpha \beta ; \alpha^{\prime} \beta^{\prime}}(\vec{x}+\vec{\Delta}, \vec{y}+\vec{\Delta}, t)$, where $\vec{\Delta}$ denotes an arbitrary 3 dimensional vector. (ii) The cubic group: $G_{\alpha \beta ; \alpha^{\prime} \beta^{\prime}}(\vec{x}, \vec{y}, t)=S_{\alpha \tilde{\alpha}}(g)$ $S_{\beta \tilde{\beta}}(g) G_{\tilde{\alpha} \tilde{\beta} ; \tilde{\alpha}^{\prime} \tilde{\beta}^{\prime}}\left(g^{-1} \vec{x}, g^{-1} \vec{y}, t\right) S_{\tilde{\alpha}^{\prime} \alpha^{\prime}}\left(g^{-1}\right) S_{\tilde{\beta}^{\prime} \beta^{\prime}}\left(g^{-1}\right)$, where $g$ denotes an arbitrary element of the cubic group. $S(g)$ denotes the (double-valued) representation matrix of $\mathrm{SO}(3)$ in the Dirac bispinor space, i.e., $S(g) \equiv \exp \left(\frac{i}{4} \sigma_{i j} \omega_{i j}\right)$ with $\sigma_{i j} \equiv-\frac{i}{2}\left[\gamma_{i}, \gamma_{j}\right]$ for $g=e^{\omega}$ with $\omega \in \operatorname{so}(3)$. (iii) The spatial reflection: $G_{\alpha \beta ; \alpha^{\prime} \beta^{\prime}}(\vec{x}, \vec{y}, t)=\left(\gamma_{0}\right)_{\alpha \tilde{\alpha}}\left(\gamma_{0}\right)_{\beta \tilde{\beta}} G_{\tilde{\alpha} \tilde{\beta} ; \tilde{\alpha}^{\prime} \tilde{\beta}^{\prime}}(-\vec{x},-\vec{y}, t)\left(\gamma_{0}\right)_{\tilde{\alpha}^{\prime} \alpha^{\prime}}\left(\gamma_{0}\right)_{\tilde{\beta}^{\prime} \beta^{\prime}}$. (iv) The charge conjugation and time-reversal: $G_{\alpha \beta ; \alpha^{\prime} \beta^{\prime}}(\vec{x}, \vec{y}, t)=\left(-C \gamma_{0}\right)_{\alpha \tilde{\alpha}}\left(-C \gamma_{0}\right)_{\beta \tilde{\beta}} G_{\tilde{\alpha} \tilde{\beta} ; \tilde{\alpha}^{\prime} \tilde{\beta}^{\prime}}^{*}(\vec{x}, \vec{y},-t)\left(-C \gamma_{0}\right)_{\tilde{\alpha}^{\prime} \alpha^{\prime}}$ $\left(-C \gamma_{0}\right)_{\tilde{\beta}^{\prime} \beta^{\prime}}$. Note that QCD Lagrangian has the charge conjugation symmetry: $\mathscr{C} q^{\mathscr{C}}{ }^{-1} \equiv C \bar{q}^{T}$, $\mathscr{C} \bar{q} \mathscr{C}^{-1} \equiv q^{T} C, \mathscr{C} A_{\mu} \mathscr{C}^{-1} \equiv-A_{\mu}^{T}$, where the matrix notation of the gluon field is adopted as $A_{\mu} \equiv A_{\mu}^{a} T^{a}$ for color SU(3) generator $T^{a}$. This implies the charge conjugation of the composite nucleon fields $\mathscr{C} N \mathscr{C}^{-1} \equiv-C \bar{N}^{T}$ and $\mathscr{C} \bar{N} \mathscr{C}^{-1}=-N^{T} C$, where $N$ represents an interpolating field for proton or neutron. Note that, for correlators in imaginary time, the charge conjugation is combined with the time-reversal through the complex conjugation.

The BS wave function $\psi_{\alpha \beta}(\vec{r})$ for the ground state is obtained from the Euclidean four point correlator in the large $t$ region after multiplied by $\mathbf{P}_{\alpha^{\prime} \beta^{\prime}}\left(J^{P}, M\right)$ as

$$
G_{\alpha \beta ; \alpha^{\prime} \beta^{\prime}}(\vec{x}, \vec{y}, t) \mathbf{P}_{\alpha^{\prime} \beta^{\prime}}\left(J^{P}, M\right)=A_{0} \psi_{\alpha \beta}\left(\vec{x}-\vec{y} ; J^{P}, M\right) e^{-E_{0} t}+\cdots,
$$

where $\mathbf{P}_{\alpha^{\prime} \beta^{\prime}}\left(J^{P}, M\right) \equiv\left(\sigma_{2}\right)_{\alpha^{\prime} \beta^{\prime}}$ and $\left(\sigma_{2} \sigma_{M}\right)_{\alpha^{\prime} \beta^{\prime}}$ for $J^{P}=0^{+}$and $1^{+}$, respectively. “..." represents contributions from excited states, which are exponentially suppressed in the large $t$ region.
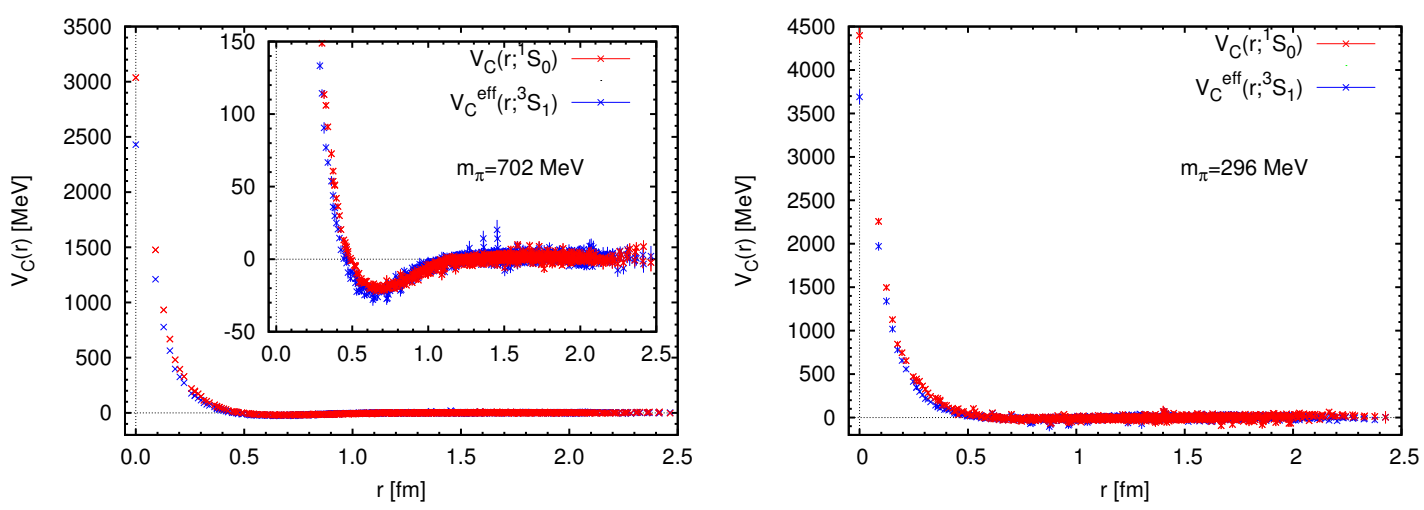

Figure 3: Full QCD results of (effective) central potentials. The 1.h.s shows the results for $\left(\kappa_{u d}, \kappa_{s}\right)=$ $(0.13700,0.13640)$, and the r.h.s. shows the results for $\left(\kappa_{u d}, \kappa_{s}\right)=(0.13770,0.13640)$, where the inset is suppressed because of the huge error bar.

Fig. 3(left) shows the full QCD results of the central force $V_{\mathrm{C}}(r) \equiv\left(E-H_{0}\right) \psi\left(\vec{x} ;{ }^{1} S_{0}\right) / \psi\left(\vec{x} ;{ }^{1} S_{0}\right)$ for ${ }^{1} S_{0}$ channel and the effective central force $V_{\mathrm{C}}^{\text {eff }}\left(r ;{ }^{3} S_{1}\right) \equiv\left(E-H_{0}\right) \psi\left(\vec{x} ;{ }^{3} S_{1}\right) / \psi\left(\vec{x} ;{ }^{3} S_{1}\right)$ for ${ }^{3} S_{1}$ channel for $\left(\kappa_{u d}, \kappa_{s}\right)=(0.13700,0.13640)$. Here, $\psi\left(\vec{x},{ }^{3} S_{1}\right)$ denotes $\psi\left(\vec{x}^{3}{ }^{3} S_{1}\right) \equiv[\mathscr{P} \psi](\vec{x})$ for notational simplicity. $V_{\mathrm{C}}\left(r ;{ }^{1} S_{0}\right)$ and $V_{\mathrm{C}}^{\text {eff }}\left(r ;{ }^{3} S_{1}\right)$ are obtained from BS wave functions on the time-slices $t=8$ and $t=9$, respectively, where the ground state saturations are achieved within error bars. Similar to the quenched results[5, 8], they have attractive pockets of about $30 \mathrm{MeV}$ in the medium distance, i.e., $0.5 \lesssim r \lesssim 1.0 \mathrm{fm}$. In contrast, the repulsive cores are considerably strong. They are by about 10 times as strong as the quenched result for comparable pion mass [8]. There seem 
to be several possible reasons. (i) A dynamical quark effect, (ii) The action adopted in quenched calculation may not be close to the continuum limit.

Fig. 3(right) shows the full QCD results of the (effective) central potential for $\left(\kappa_{u d}, \kappa_{s}\right)=$ $(0.13770,0.13640)$. These results are obtained from the BS wave functions on the time-slice $t=6$, where the ground state saturations are achieved within the statistical errors. We do not show the inset because of huge statistical errors. Although it is necessary to improve the statistics significantly to reduce the huge error bars, we see that the repulsive cores are again considerably strong.

\section{Summary}

We have presented preliminary lattice QCD results for the tensor force by using quenched QCD. We have seen that the tensor force has a large quark mass dependence and is enhanced as the quark mass decreases. We have presented preliminary results of the (effective) central force from $2+1$ flavor lattice QCD by using PACS-CS gauge configurations. A remarkable difference from the quenched results was found in the strength of the repulsive core. It is interesting to investigate the reason, since it may provide us with a key to the origin of the repulsive core, which is one of the most important open problems in the nuclear physics.

\section{Acknowledgments}

Quenched QCD Monte Carlo calculations have been done with IBM Blue Gene/L at KEK under a support of its Large Scale simulation Program Nos. 06-21, 07-07, 08-19. The full QCD calculations have been done with PACS-CS under the "Interdisciplinary Computational Science Program" of Center for Computational Sciences, University of Tsukuba (No 08a-12). We thank PACS-CS Collaboration for the $2+1$ flavor gauge configurations. We are grateful for authors and maintainers of $C P S++[10]$, of which a modified version is used for measurement done in this work. N.I. thanks Dr. T. Izubuchi for a sample code of 3D FFT. This work was supported in part by Grant-in-Aid of the Japanese Ministry of Education, Science, Support and Culture (Nos. 18540253, 19540261, 20340047).

\section{References}

[1] H. Yukawa, Proc. Math. Phys. Soc. Japan 17, 48 (1935).

[2] M. Taketani et al., Prog. Theor. Phys. Suppl. 39,1 (1967); 42, 1 (1968).

[3] T. Jastrow, Phys. Rev. 81, 165 (1951).

[4] T.T. Takahashi, T. Doi, H. Suganuma, AIP Conf. Proc. 842, 249 (2006).

[5] N. Ishii, S. Aoki, T. Hatsuda, Phys. Rev. Lett. 99, 022001 (2007).

[6] S. Aoki, T. Hatsuda, N. Ishii, arXiv:0805.2462[hep-ph].

[7] M. Fukugita, Y. Kuramashi, M. Okawa, H. Mino, A. Ukawa, Phys. Rev. D52, 3003 (1995).

[8] N. Ishii, S. Aoki, T. Hatsuda, PoS(LAT2007), 146(2007).

[9] PACS-CS Collaboration(S. Aoki et al.), arXiv:0807.1661[hep-lat].

[10] CPS++ http://qcdoc.phys.columbia.edu/chuiwoo_index.html (maintainer: Chulwoo Jung). 